\title{
Perturbative thresholds in the physical region
}

\author{
Antonio O. Bouzas* \\ Departamento de Física Aplicada, CINVESTAV-IPN \\ Apdo. Postal 73 "Cordemex," Mérida 97310, Yucatán, México
}

\begin{abstract}
We consider unstable-particle scattering in the context of 3-body processes. We show that all partial-wave cross-sections are finite and positive, and the total cross-section is proportional to the transverse size of space in the region of on-shell particle exchange. We comment on the role of loop corrections.
\end{abstract}

\section{Introduction.}

At tree-level, Feynman diagrams for 2-body scattering processes can have just simple poles as singularities in the physical region. In particular, logarithmic thresholds only appear in higherorder diagrams. The singularity structure of Feynman graphs becomes increasingly complicated as the number of external legs grows. Thus, we can have thresholds in the physical region of tree-level graphs if the number of external legs is larger than four. By physical region we understand here real, on-shell external momenta.

Consider, for instance, the 3-body tree-level graphs in Figs. (1a) and (1b). For concreteness, we shall temporarily assume that these are QED Feynman graphs. In both diagrams the two upper vertices can be viewed as a 2-body scattering process one of whose final-state particles subsequently undergoes a further 2-body subprocess comprising the two lower vertices. Clearly, there is a domain of external momenta in the physical region for these graphs where the internal particle shared by both scattering subprocesses is kinematically allowed to be on its mass shell. As functions of the squared center-of-mass energy $s$, these Feynman graphs have a branch-cut extending over the interval of values of $s$ for which the on-shell propagation of the internal fermion is kinematically possible. Such singularity is not present in graph (1c), unless the external photon lines are sufficiently off-shell.

There is another situation in which such singularity can occur in a 2-body scattering diagram. Imagine that the wavy lines in (1a) and (1b) are not photons but unstable massive bosons that can decay into an $e^{+} e^{-}$pair. If the momenta of the external particles connected to these bosons in (1a) were chosen appropiately, the momentum circulating along boson lines would lie on their resonance peak and diagram (1a) could dominate over (1b) and other 3-body diagrams contributing to the same amplitude. In this kinematic region, then, the process would be effectively 2-body, and could be represented by graph (1c) — with the caveat, though, that (1c) is only a short-hand notation for (1a), since unstable particles cannot be asymptotic states.

*E-mail: abouzas@fis.cinvestav.mx 
If the external unstable particles in (1c) were long-lived enough, such scattering process could be accesible to experiments. We would then be interested in computing the cross-section of a 2-body process possessing the type of singularities discussed above. This problem has been posed before, in connection with several different phenomenological situations. For example, the process $N^{*} \pi \rightarrow N^{*} \pi$, in which the intermediate particle is a nucleon, was considered in [1] in relation with the so-called "Peierl's mechanism." The process $\mu^{+} \mu^{-} \rightarrow W W^{*}$ was considered in [2], and a similar problem was discussed in the context of an effective model Lagrangian in [3]. In all of these cases the amplitudes for graphs analogous to (1c) were assumed to have a Breit-Wigner form, in spite of the fact that the exchanged particle is stable. Such assumption can be heuristically motivated by the fact that the external unstable states are on their "mass-shell," which is identified with their complex pole mass [1]. In the familiar case of resonance-formation processes in the $s$-channel, the Breit-Wigner form of the amplitude can be rigorously proved by Dyson resummation of the propagator followed by Laurent expansion around the pole [4. As far as we know, no proof exists in the case of processes like $(1 \mathrm{a}, \mathrm{b}, \mathrm{c})$. Below we shall argue that this assumption can lead to a good approximation to the tree-level cross-section in some situations.

The singularity in 3-body diagrams like (1a) and (1b) is a manifestation of a well-known phenomenon, namely, that a singularity in an amplitude occurs if the interaction is unbounded in space-time. As long as the exchanged particle in $(1 \mathrm{a}, \mathrm{b}, \mathrm{c})$ is virtual the uncertainty principle restricts the space-time distance over which it can propagate, but when it can be on-shell the process becomes unbounded and the amplitude singular [5, §18.5]. The same situation can occur in higher-order diagrams. According to a theorem by Coleman and Norton [6] (see also [0, §6.3.4]), a Feynman graph will possess a singularity in its physical region if and only if the graph can be interpreted as a momentum-conserving process in space-time with all internal lines on their massshell and propagating forwards in time円. The Coleman-Norton theorem is formulated for 1-particle irreducible diagrams and valid to all orders of perturbation theory. The singularities in tree-level graphs such as $(1 \mathrm{a}, \mathrm{b})$ have the same physical origin.

In the particular situation considered here of graphs (1a,b,c) these general statements can be made more quantitative. The physical region for $s$ in $(1 \mathrm{a}, \mathrm{b})$ is of the form $0<s_{\mathrm{th}} \leq s<\infty$. The kinematical region over which on-shell internal particles are allowed will be limited by values $s_{ \pm}$, and will partially or completely overlap the physical region. Let us assume, for concreteness, that $s_{\text {th }}<s_{-}<s_{+}$. In the region $s_{-} \leq s \leq s_{+}$where the internal particle can be on-shell, it can propagate over arbitrarily long distances. Therefore, although the largest momentum it can have is finite, the angular momentum of the process can be arbitrarily large. In fact, an on-shell internal particle will feed partial waves with essentially uniform probability, leading to a total cross-section that behaves as,

$$
\sigma=\sum_{\ell=0}^{L} \sigma^{\ell} \sim \sum_{\ell=0}^{L} \mathcal{O}(1) \sim \mathcal{O}(L) \quad \text { as } \quad L \rightarrow \infty .
$$

Since $L \sim p b$ with $p$ the (fixed) transverse linear momentum and $b$ the impact parameter, we conclude that the cross-section due to on-mass-shell particle exchange diverges as $\sigma \sim b$, where $b$ is the transverse dimension of space.

On the other hand, in the region $s<s_{-}$or $s>s_{+}$, the interaction region is bounded. Each partial-wave cross-section $\sigma^{\ell}$ is finite and decreases rapidly with $\ell$ so that the sum converges to a finite value $\sigma$. As $s$ approaches $s_{-}\left(s_{+}\right)$from below (above), the intermediate particle can be closer

\footnotetext{
${ }^{1}$ Notice, however, that the notion of "physical region" used in [6] is less restrictive than ours. External lines are not required to be on-shell in [6].
} 
to its mass-shell and propagate over increasingly larger distances. Even though for each partial wave the transverse size of the process is fixed, since $\ell$ is fixed, in the limit $s \rightarrow s_{ \pm}$the longitudinal size is unbounded and as a consequence each partial wave is singular at $s=s_{ \pm}$. The singularity is logarithmic, though, and therefore integrable, thus leading to finite results for suitably averaged observables.

We substantiate these assertions in the rest of this paper. In the next section we perform a brief kinematical analysis which serves to establish our notation. We also define there the cross-section for graph (1c), which is derived from (1a). In section 3 we show that all partial-wave cross-sections are finite and positive and, as shown in section 4, they result in a total cross-section wich diverges with the transverse dimension of space as indicated above. In section 5 we give some final remarks, commenting in particular on the rôle of loop corrections.

\section{2- and 3-body processes.}

We consider in this section the situation described in the Introduction, in which diagrams of the type (1a) dominate over other diagrams contributing to the amplitude. We will work with scalar fields for simplicity, with couplings of the form $g \phi^{\dagger} \phi X$, where $\phi$ is a light complex scalar field and $X$ a heavy real one with $m_{X}>2 m_{\phi}$. We then have the graphs shown in figure 2 for the process,

$$
\left(\phi\left(p_{1}\right) \bar{\phi}\left(p_{2}\right)\right) \phi\left(k_{1}\right) \rightarrow X\left(q_{1}\right) \phi\left(k_{1}\right) \rightarrow Y\left(q_{2}\right) \phi\left(k_{2}\right) \rightarrow\left(\phi\left(p_{3}\right) \bar{\phi}\left(p_{4}\right)\right) \phi\left(k_{2}\right) .
$$

Here we have a different scalar $Y$ in the final state, with mass $m_{Y}>2 m_{\phi}$ and coupling $g \phi^{\dagger} \phi Y$, but we shall not introduce a new coupling constant for simplicity. We assume that the initial stable particles are described by plane waves and therefore have sharply defined momenta. Furthermore, $q_{1}=p_{1}+p_{2}$ and $q_{2}=p_{3}+p_{4}$ are assumed to lie within the resonance peak of $X$ and $Y$, respectively.

We introduce Mandelstam invariants in the usual way, by reference to the underlying 2-body processes,

$$
s=\left(q_{1}+k_{1}\right)^{2} ; \quad t=\left(k_{2}-k_{1}\right)^{2} ; \quad u=\left(k_{2}-q_{1}\right)^{2} .
$$

We then have,

$$
\begin{aligned}
\mathcal{M}_{3 \rightarrow 3} & =\frac{-g}{q_{1}^{2}-m_{X}^{2}+i m_{X} \Gamma_{X}} \mathcal{M}_{2 \rightarrow 3} \\
\mathcal{M}_{2 \rightarrow 3} & =\frac{-g}{q_{2}^{2}-m_{Y}^{2}+i m_{Y} \Gamma_{Y}} \mathcal{M}_{2 \rightarrow 2} \\
\mathcal{M}_{2 \rightarrow 2} & =-i g^{2}\left\{\frac{1}{u-m_{\phi}^{2}+i 0^{+}}+\frac{1}{s-m_{\phi}^{2}+i 0^{+}}\right\}
\end{aligned}
$$

We retained only the pole part in the $X$ and $Y$ propagators, in view of our assumption that their momenta are close to the peak. Since $q_{1}$ remains constant as we vary the final state momenta, we defined an amplitude $\mathcal{M}_{2 \rightarrow 3}$ for the process with an unstable $X$ particle in the initial state by factoring out the constant propagator and production vertex. We also defined a 2-body amplitude $\mathcal{M}_{2 \rightarrow 2}$ by formally applying Feynman rules to the 2-body process.

The $s$-variable physical region for the 3 -body scattering is $s \geq 9 m_{\phi}^{2}$. Since we are interested in the kinematic region where both $X$ and $Y$ are close to their resonance peak, we must have,

$$
s \geq s_{\text {th }}=\left(\max \left(m_{X}, m_{Y^{*}}\right)+m_{\phi}\right)^{2}
$$


where we denoted $q_{2}^{2}=\left(p_{3}+p_{4}\right)^{2}=m_{Y^{*}}^{2}$, with $m_{Y^{*}}$ close to $m_{Y}$. We let $q_{1}^{2}=m_{X}^{2}$ exactly for concreteness, although it could as well have another value within $\sim \Gamma_{X}$ of $m_{X}$.

Given $q_{1}^{2}=m_{X}^{2}$ and $q_{2}^{2}=m_{Y^{*}}^{2}$, at fixed $s$ the squared-momentum flowing through the internal $\phi$ line is restricted to the interval $u_{-} \leq u \leq u_{+}$with

$$
\begin{aligned}
u_{ \pm}= & \frac{m_{X}^{2}+m_{Y^{*}}^{2}+2 m_{\phi}^{2}-s}{2}+\frac{\left(m_{X}^{2}-m_{\phi}^{2}\right)\left(m_{Y^{*}}^{2}-m_{\phi}^{2}\right)}{2 s} \pm \\
& \pm \frac{1}{2 s} \sqrt{\left(s-m_{X}^{2}+m_{\phi}^{2}\right)^{2}-4 s m_{\phi}^{2}} \sqrt{\left(s-m_{Y^{*}}^{2}+m_{\phi}^{2}\right)^{2}-4 s m_{\phi}^{2}} .
\end{aligned}
$$

The singularity at $u=m_{\phi}^{2}$ in the amplitude falls in the physical region if $u_{-} \leq m_{\phi}^{2} \leq u_{+}$. From (8) we see that this is possible only when $s_{-} \leq s \leq s_{+}$, where

$$
s_{ \pm}=\frac{m_{X}^{2} m_{Y^{*}}^{2}+2 m_{\phi}^{4} \pm m_{X} m_{Y^{*}} \sqrt{m_{X}^{2}-4 m_{\phi}^{2}} \sqrt{m_{Y^{*}}^{2}-4 m_{\phi}^{2}}}{2 m_{\phi}^{2}} .
$$

Notice that $u_{+} \leq m_{X}^{2}, m_{Y}^{2}$ as long as $s \geq s_{\text {th }}$, and that $s_{-} \geq s_{\text {th }}$, so that the region where exchange of an on-shell $\phi$ is kinematically allowed is entirely within the physical region of the 2-body process, but the absorptive part of the $\phi$ propagator vanishes in that region. The range of variation of $t$ and its values in the region $s_{-} \leq s \leq s_{+}$can be obtained from $s+t+u=m_{X}^{2}+m_{Y^{*}}^{2}+2 m_{\phi}^{2}$.

In what follows we shall assume $m_{X}, m_{Y} \gg m_{\phi}$ for simplicity. In that case we get,

$$
u_{-}=m_{X}^{2}+m_{Y^{*}}^{2}-s ; \quad u_{+}=\frac{m_{X}^{2} m_{Y^{*}}^{2}}{s} ; \quad u_{+}-u_{-}=\frac{\left(s-m_{X}^{2}\right)\left(s-m_{Y^{*}}^{2}\right)}{s}
$$

instead of the more complicated expressions (8), and

$$
s_{-}=m_{X}^{2}+m_{Y^{*}}^{2} ; \quad s_{+}=\frac{m_{X}^{2} m_{Y^{*}}^{2}}{m_{\phi}^{2}} \rightarrow+\infty .
$$

Cross-sections for the $2 \rightarrow 3$ process are given by,

$$
\sigma_{2 \rightarrow 3}=\frac{1}{2\left(s-m_{X}^{2}\right)} \int \frac{d^{3} k_{2}}{(2 \pi)^{3} 2 k_{2}^{0}} \frac{d^{3} p_{3}}{(2 \pi)^{3} 2 p_{3}^{0}} \frac{d^{3} p_{4}}{(2 \pi)^{3} 2 p_{4}^{0}}(2 \pi)^{4} \delta\left(P_{\text {Tot }}-k_{2}-p_{3}-p_{4}\right) \Theta(\text { cuts })\left|\mathcal{M}_{2 \rightarrow 3}\right|^{2}
$$

$P_{\text {Tot }}$ being the total 4-momentum of the process, and $\Theta$ (cuts) a step function enforcing the cuts that define the cross-section we are computing. We shall only use a loose lower bound on $m_{Y^{*}}^{2}$ as cut in this paper, for reasons to be explained below. $\sigma_{2 \rightarrow 3}$ is conventionally defined in (12) in terms of the flux-factor corresponding to an initial $|X, \phi\rangle$ state.

In order to express $\sigma_{2 \rightarrow 3}$ as a function of $q_{2}$ we make a change of variables, $q_{2}=p_{3}+p_{4}, \Delta=$ $p_{4}-p_{3}$, and integrate over $\Delta$ to obtain,

$$
\sigma_{2 \rightarrow 3}=\frac{g^{2}}{8(2 \pi)^{4}\left(s-m_{X}^{2}\right)} \int d^{4} k_{2} d^{4} q_{2} \delta_{+}\left(k_{2}^{2}\right) \delta\left(P_{\text {Tot }}-k_{2}-q_{2}\right) \frac{\Theta(\text { cuts })}{\left(q_{2}^{2}-m_{Y}^{2}\right)^{2}+m_{Y}^{2} \Gamma_{Y}^{2}}\left|\mathcal{M}_{2 \rightarrow 2}\right|^{2},
$$

in terms of $\mathcal{M}_{2 \rightarrow 2}$. Since $q_{2}$ is kinematically guaranteed to be in the forward light-cone, a resolution of the identity exists,

$$
1=\int_{0}^{\infty} d\left(m_{Y^{*}}^{2}\right) \delta\left(q_{2}^{2}-m_{Y^{*}}^{2}\right)
$$


which, upon insertion in $\sigma_{2 \rightarrow 3}$ and integration over $q_{2}$ and $k_{2}^{0},\left|\mathbf{k}_{2}\right|$, yields,

$$
\sigma_{2 \rightarrow 3}=\frac{g^{2}}{(2 \pi)^{3} 64 s} \int_{-1}^{1} d(\cos \theta) \int_{0}^{s} d\left(m_{Y^{*}}^{2}\right) \frac{\Theta(\mathrm{cuts})}{\left(m_{Y^{*}}^{2}-m_{Y}^{2}\right)^{2}+m_{Y}^{2} \Gamma_{Y}^{2}} \frac{s-m_{Y^{*}}^{2}}{s-m_{X}^{2}}\left|\mathcal{M}_{2 \rightarrow 2}\right|^{2}
$$

where $\theta$ is the scattering angle, $\cos \theta \propto \mathbf{k}_{1} \cdot \mathbf{k}_{2}$.

We can now define a 2-body cross-section by factoring out the coupling constant and phasespace factor coming from the $Y$ decay vertex,

$$
\begin{aligned}
\sigma_{2 \rightarrow 2} & =\frac{1}{32 \pi s} \int_{-1}^{1} d(\cos \theta) \int_{0}^{s} d\left(m_{Y^{*}}^{2}\right) \Theta(\text { cuts }) \Delta_{B W}\left(m_{Y^{*}}^{2}\right) \frac{s-m_{Y^{*}}^{2}}{s-m_{X}^{2}}\left|\mathcal{M}_{2 \rightarrow 2}\right|^{2} \\
\Delta_{B W}\left(m_{Y^{*}}^{2}\right) & \equiv \frac{m_{Y} \Gamma_{Y} / \pi}{\left(m_{Y^{*}}^{2}-m_{Y}^{2}\right)^{2}+m_{Y}^{2} \Gamma_{Y}^{2}} .
\end{aligned}
$$

This equation can be rewritten in the more suggestive form,

$$
\sigma_{2 \rightarrow 2}=\int_{0}^{s} d\left(m_{Y^{*}}^{2}\right) \Theta(\text { cuts }) \Delta_{B W}\left(m_{Y^{*}}^{2}\right) \widetilde{\sigma}_{2 \rightarrow 2},
$$

where $\widetilde{\sigma}_{2 \rightarrow 2}$ is the cross-section we would obtain by pretending that $X$ and $Y$ are asymptotic states and applying the Feynman rules to the 2-body process directly. Clearly, the same equation holds mutatis-mutandis for the differential cross-section $d \sigma_{2 \rightarrow 2} / d(\cos \theta)$. From (6) we immediately obtain for $\widetilde{\sigma}_{2 \rightarrow 2}$,

$$
\tilde{\sigma}_{2 \rightarrow 2}=\frac{g^{4}}{16 \pi s} \frac{s-m_{Y^{*}}^{2}}{s-m_{X}^{2}}\left\{\frac{1}{s^{2}}+\frac{s}{m_{X}^{2} m_{Y^{*}}^{2}\left(s_{-}-s\right)}+\frac{2}{\left(s-m_{X}^{2}\right)\left(s-m_{Y^{*}}^{2}\right)} \ln \left(\frac{m_{X}^{2} m_{Y^{*}}^{2}}{s} \frac{1}{s_{-}-s}\right)\right\} .
$$

For completeness, we also quote the form of $\widetilde{\sigma}_{2 \rightarrow 2}$ when we do not neglect $m_{\phi}$ in comparison with $m_{X, Y}$,

$$
\widetilde{\sigma}_{2 \rightarrow 2}=\frac{g^{4}}{16 \pi s} \frac{\left|\mathbf{k}_{2}\right|}{\left|\mathbf{k}_{1}\right|}\left\{\frac{1}{\left(s-m_{\phi}\right)^{2}}+\frac{s}{m_{\phi}^{2}} \frac{1}{\left(s-s_{-}\right)\left(s-s_{+}\right)}+\frac{1}{2} \frac{1}{\left|\mathbf{k}_{2}\right|\left|\mathbf{k}_{1}\right|\left(s-m_{\phi}^{2}\right)} \ln \left(\frac{u_{+}(s)-m_{\phi}^{2}}{u_{-}(s)-m_{\phi}^{2}}\right)\right\},
$$

where $\left|\mathbf{k}_{2}\right|$ refers to its center-of-mass frame value, and $u_{ \pm}$as functions of $s$ are given in (8). This expression for the cross-section is valid for $s_{\text {th }} \leq s<s_{-}$and $s>s_{+}$, with $s$ "far" (to be quantified below) from the singular points $s_{ \pm}$. In the interval between $s_{ \pm}$the direct $u$-channel contribution is negative and unbounded, and the $s$ - $u$ interference term becomes imaginary.

\section{Partial-Wave amplitudes and cross-sections.}

The amplitude $\mathcal{M}_{2 \rightarrow 2}$ (see (66) ) entering the 2-body cross-section defined in (16) can be expanded in partial waves,

$$
\mathcal{M}_{2 \rightarrow 2}=-i g^{2}\left(\frac{1}{s+i 0^{+}}+\frac{2}{u_{+}-u_{-}} \sum_{\ell=0}^{\infty}(2 \ell+1) Q_{\ell}\left(\xi+i 0^{+}\right) P_{\ell}(\cos \theta)\right),
$$

where $u_{ \pm}$are defined in (10), $P_{\ell}$ are Legendre polynomials and $Q_{\ell}$ Legendre functions of the second kind [8, 9], $\theta$ is the scattering angle and,

$$
\xi=1+\frac{2 u_{-}}{u_{+}-u_{-}}=1+\frac{2 s\left(m_{X}^{2}+m_{Y^{*}}^{2}-s\right)}{\left(s-m_{X}^{2}\right)\left(s-m_{Y^{*}}^{2}\right)} .
$$


$Q_{\ell}(z)$ has a logarithmic branch-cut for $z$ real, $-1 \leq z \leq 1$, so we see that each partial-wave amplitude has a branch-cut in the region of on-shell $\phi$ exchange, $s_{-} \leq s<s_{+}\left(\rightarrow+\infty\right.$ as $\left.m_{\phi} / m_{X, Y} \rightarrow 0\right)$. Notice that in the case of a genuine 2-body scattering the partial-wave expansion (21,22) would hold unchanged, but we would have $\xi>1$ for all finite $s$ in the physical region. Only in 3-body processes can $\xi$ go through 1, and in that case there is on-shell particle exchange.

For $s_{\text {th }} \leq s<s_{-}$we must take the principal branch of $Q_{\ell}$, i.e. $Q_{\ell}(\xi>1)$ real. Therefore, as $s$ goes through $s_{-}$, and $\xi$ goes through 1 , the $i 0^{+}$prescription results in,

$$
Q_{\ell}\left(\xi+i 0^{+}\right)=Q_{\ell}(\xi)-i \frac{\pi}{2} P_{\ell}(\xi)
$$

with $Q_{\ell}$ a Legendre function "on the cut" [8, 9]. The first term in (23) gives the dispersive, and the second the absorptive, part of the $u$-channel amplitude $\left(u+i 0^{+}\right)^{-1}$. Therefore, they refer to "virtual" and "real," or on-shell, particle exchange, respectively.

We shall separate the contributions to the cross-section coming from the regions $s<s_{-}$and $s>s_{-}$for convenience,

$$
\sigma_{2 \rightarrow 2}=\sum_{\ell=0}^{\infty} \sigma_{2 \rightarrow 2}^{\ell} ; \quad \sigma_{2 \rightarrow 2}^{\ell}=\left(\sigma_{2 \rightarrow 2}^{\ell}\right)_{B}+\left(\sigma_{2 \rightarrow 2}^{\ell}\right)_{A},
$$

where the contributions from the regions "below" and "above" $s_{-}$are found by substituting each squared partial-wave amplitude in expression (16) for the cross-section. Thus, for $\ell>0$ we have,

$$
\begin{aligned}
& \left(\sigma_{2 \rightarrow 2}^{\ell>0}\right)_{B}=\frac{(2 \ell+1) g^{4} s}{4 \pi\left(s-m_{X}^{2}\right)^{3}} \int_{s-m_{X}^{2}}^{s} d\left(m_{Y^{*}}^{2}\right) \frac{\Theta(\text { cuts }) \Delta_{B W}\left(m_{Y^{*}}^{2}\right)}{s-m_{Y^{*}}^{2}} Q_{\ell}^{2}(\xi) \\
& \left(\sigma_{2 \rightarrow 2}^{\ell>0}\right)_{A}=\frac{(2 \ell+1) g^{4} s}{4 \pi\left(s-m_{X}^{2}\right)^{3}} \int_{0}^{s-m_{X}^{2}} d\left(m_{Y^{*}}^{2}\right) \frac{\Theta(\text { cuts }) \Delta_{B W}\left(m_{Y^{*}}^{2}\right)}{s-m_{Y^{*}}^{2}}\left(\mathrm{Q}_{\ell}^{2}(\xi)+\frac{\pi^{2}}{4} P_{\ell}^{2}(\xi)\right)
\end{aligned}
$$

and for $\ell=0$, with the contribution from the $s$-channel diagram in figure $(2 \mathrm{~b})$,

$$
\begin{aligned}
\sigma_{2 \rightarrow 2}^{\ell=0}= & \frac{g^{4}}{16 \pi s} \int_{0}^{s} d\left(m_{Y^{*}}^{2}\right) \Theta(\text { cuts }) \Delta_{B W}\left(m_{Y^{*}}^{2}\right) \frac{s-m_{Y^{*}}^{2}}{s-m_{X}^{2}} \times \\
& \times\left\{\frac{1}{s^{2}}+\frac{4}{\left(s-m_{X}^{2}\right)\left(s-m_{Y^{*}}^{2}\right)}\left(\Theta(\xi-1) Q_{0}(\xi)+\Theta(1-\xi) \mathrm{Q}_{0}(\xi)\right)\right. \\
& \left.+\frac{4 s^{2}}{\left(s-m_{X}^{2}\right)^{2}\left(s-m_{Y^{*}}^{2}\right)^{2}}\left(\Theta(\xi-1) Q_{0}^{2}(\xi)+\Theta(1-\xi)\left(\mathrm{Q}_{0}^{2}(\xi)+\frac{\pi^{2}}{4}\right)\right)\right\},
\end{aligned}
$$

where $\xi$ as a function of $m_{Y^{*}}^{2}, m_{X}^{2}$ and $s$ is given in (22), and we used (10). We could also write $\sigma_{2 \rightarrow 2}$ as a sum of "virtual" and "real" contributions, obtained from (24 27) by setting $P_{\ell} \equiv 0$ or $Q_{\ell} \equiv 0 \equiv \mathrm{Q}_{\ell}$, respectively.

There are three potentially dangerous singularities in the integrands of (25 27). In the first place, terms involving $Q_{\ell}$ are integrated over $s-m_{X}^{2} \leq m_{Y^{*}}^{2} \leq s$, so that the factor $\left(s-m_{Y^{*}}^{2}\right)^{-1}$ is singular at the upper end of integration. Since $\xi=\mathcal{O}\left(1 /\left(s-m_{Y^{*}}^{2}\right)\right)$ as $m_{Y^{*}}^{2} \rightarrow s$, however, that would-be pole is cancelled by the zero of order $\ell+1$ of $Q_{\ell}$ at infinity.

Secondly, (25 27) are all singular at $s \rightarrow\left(m_{X}^{2}\right)^{+}$. These are spurious singularities due to our neglecting $m_{\phi}$. Let us consider those integrals involving $Q_{\ell \geq 0}$ first. As long as $m_{Y^{*}}^{2}>0$, we have $\xi=\mathcal{O}\left(1 /\left(s-m_{X}^{2}\right)\right)$ as $s \rightarrow\left(m_{X}^{2}\right)^{+}$, so the singularity is cancelled by the zero of $Q_{\ell}$ at infinity. 
If $m_{Y^{*}}^{2}=0$, however, $\xi$ remains finite as $s \rightarrow\left(m_{X}^{2}\right)^{+}$and there is a pole in that limit. We will introduce a cut in the domain of integration,

$$
\Theta(\text { cuts })=\Theta\left(m_{Y^{*}}^{2}-\lambda^{2}\right)
$$

with $\lambda^{2}>0$, so the singularity does not develop. We will elaborate further on the physical meaning of this kinematical cut below. Notice, however, that each partial-wave cross-section should be insensitive to the precise value of $\lambda^{2}$, at least so long as $\Gamma_{Y} \ll m_{Y}$. If $\Gamma_{Y} \sim m_{Y}$, then the very notion of an effectively 2-body scattering comes into question and the whole process should be dealt with, including all possible diagrams with 3 stable particles in the final state. The cut (28) also solves the problem in the other integrals not involving $Q_{\ell}$, since now we must have $s \geq m_{X}^{2}+\lambda^{2}>m_{X}^{2}$ in order to be in the region of on-shell $\phi$-exchange, and those integrals are then strictly zero for $s<m_{X}^{2}+\lambda^{2}$.

Lastly, there is a branch point of $Q_{\ell}$ and $Q_{\ell}$ at $\xi=1$ (or, $m_{Y^{*}}^{2}=s-m_{X}^{2}$ ) which is an endpoint of integration. This is actually the only true singularity of the integrand. It is a logarithmic singularity, though, and therefore integrable. The partial-wave cross-sections (25 26) are thus finite and positive for all $\ell$ and $s \geq s_{\mathrm{th}}$.

These expressions for partial-wave cross-sections are to be compared with the heuristic Ansatz [1, 2, 3],

$$
\mathcal{M}_{2 \rightarrow 2}^{\mathrm{Ansatz}}=-i g^{2}\left(\frac{1}{u+i m_{Y} \Gamma_{Y}}+\frac{1}{s+i 0^{+}}\right),
$$

leading to partial-wave cross-sections of the form,

$$
\left(\sigma_{2 \rightarrow 2}^{\ell>0}\right)_{\text {Ansatz }}=\frac{g^{4}}{4 \pi s} \frac{1}{\left(u_{+}-u_{-}\right)^{2}} \frac{s-m_{Y}^{2}}{s-m_{X}^{2}}(2 \ell+1)\left|Q_{\ell}\left(\tilde{\xi}+\frac{2 i m_{Y} \Gamma_{Y}}{u_{+}-u_{-}}\right)\right|^{2},
$$

where $\tilde{\xi}$ is given by (22) with $m_{Y^{*}}=m_{Y}$. Both (25 26) and (30) approach (19) in the narrow resonance limit $\Gamma_{Y} / m_{Y} \rightarrow 0$, and depend on $\Gamma_{Y}$ as $\ln ^{2}\left(\Gamma_{Y}\right)$ at the maximum at $s=s_{-}$. Therefore, we expect (25 26) and (30) to be close to each other for moderate values of $\Gamma_{Y} / m_{Y}$ and $\ell$. We notice, however, that (30) leads to a finite total cross-section over the whole kinematical range $s \geq s_{\text {th }}$, so that it must have a different $\ell$-dependence for large values $\ell \gtrsim m_{Y} / \Gamma_{Y}$.

Before turning to total cross-sections, we would like to remark that equations (25-26) for $\sigma_{2 \rightarrow 2}^{\ell}$ were derived in the idealized assumption that the initial- $X$ momentum is sharply defined and, in particular, its squared-momentum is precisely known. Let us assume now that $q_{1}$ in figure (2) is statistically distributed, with a flat probability density $\mathcal{F}$ over a range of values centered around $q_{1}^{2}=m_{X}^{2}$. The squared amplitude in this case takes the form of an incoherent sum, schematically,

$$
\int d\left(m_{X^{*}}^{2}\right) \mathcal{F}\left(m_{X^{*}}^{2}\right) \Delta_{B W}\left(m_{X^{*}}^{2}\right) \int d\left(m_{Y^{*}}^{2}\right) \Delta_{B W}\left(m_{Y^{*}}^{2}\right)\left|\mathcal{M}_{2 \rightarrow 2}\right|^{2} .
$$

If $\mathcal{F}\left(m_{X^{*}}^{2}\right)$ is very narrow, or if $\Gamma_{X} / m_{X} \rightarrow 0$, we recover (25 27). If, instead, $\Gamma_{Y} / m_{Y} \ll \Gamma_{X} / m_{X}$, then we can replace $\Delta_{B W}\left(m_{Y^{*}}^{2}\right) \sim \delta\left(m_{Y^{*}}^{2}-m_{Y}^{2}\right)$ and we have,

$$
\int d\left(m_{X^{*}}^{2}\right) \mathcal{F}\left(m_{X^{*}}^{2}\right) \Delta_{B W}\left(m_{X^{*}}^{2}\right)\left|\mathcal{M}_{2 \rightarrow 2}\right|^{2} .
$$

If $\Delta_{B W}\left(m_{X^{*}}^{2}\right)$ is narrower than $\mathcal{F}\left(m_{X^{*}}^{2}\right)$, the latter is essentially constant over the range of integration so it can be taken outside the integral and we end up with an expression analogous to 
(25 27), but with $\Delta_{B W}\left(m_{X^{*}}^{2}\right)$ in place of $\Delta_{B W}\left(m_{Y^{*}}^{2}\right)$. In this case, the correct Ansatz is (29) with $m_{X} \Gamma_{X}$ instead of $m_{Y} \Gamma_{Y}$. A similar result obtains if we consider wave-packets as initial state rather than an incoherent superposition. In that case the methods of [10] must be applied, but we shall not go into the details here.

\section{Total cross-sections.}

A direct application of Feynman rules in the Born approximation to the graphs shown in figure 2 leads to the total cross-section (19), which is singular as $s \rightarrow s_{-}$from below and negative for $s>s_{-}$. As we have seen in the previous section, all partial-wave cross-sections $\sigma_{2 \rightarrow 2}^{\ell}$ are finite and positive. If we try to compute $\sigma_{2 \rightarrow 2}$ as the sum of partial-wave cross-sections (24), however, we are obviously led back to (18,19) as can be explicitly checked. This fact tells us that the singularities in the total cross-section are due to the bad convergence properties of the sum in (24) or, equivalently, of $\sigma_{2 \rightarrow 2}^{\ell}$ as $\ell \rightarrow \infty$. The physical reasons for this bad convergence were discussed in the Introduction. In this section we will quantitatively study the convergence properties of $\sigma_{2 \rightarrow 2}^{\ell}$. In the next two subsections we present some simple but somewhat technical results which we discuss below in subsection 4.3 .

\subsection{The region of on-shell exchange}

Let us first consider the term in $(26)$ that involves $P_{\ell}$. It can be rewritten as,

$$
\int_{-1}^{1} d \xi \varphi(\xi) P_{\ell}^{2}(\xi)
$$

where $\varphi$ represents the combination of factors in front of $P_{\ell}$ in the integrand of (26). In particular, $\varphi$ contains a factor $\Theta$ (cuts). As we will now see, the dependence of (33) on $\ell$ is not sensitive to the details of the cut, such as the precise value of $\lambda^{2}$ or the form of $\Theta$. We will take $\Theta$ not as a step-function, but as some smooth approximation to it. In this way we can consider $\varphi$ to be continuous, non-negative and not everywhere-vanishing on the interval of integration.

We will now show that $(33)$ is $\mathcal{O}(1 / \ell)$ as $\ell \rightarrow \infty$. This may seem obvious in view of the normalization of $P_{\ell}$, but the key-point here is that as long as $\varphi$ is positive and continuous the convergence will not be faster, independently of the particular form of $\varphi$. Since $\varphi$ is continuous over a compact interval, it can be uniformly approximated by a polynomial to any desired accuracy, due to a theorem of Weierstrass [11]. Therefore, we need only consider the case of polynomial $\varphi$. Since it is independent of $\ell$, we may integrate $\varphi$ term by term in (33) and study the convergence of each term separately. Furthermore, since $P_{\ell}^{2}$ is even, we need only consider even powers of $\xi$.

Thus, we have to see that,

$$
\int_{-1}^{1} d x\left(x^{n} P_{\ell}(x)\right)^{2}=\mathcal{O}\left(\frac{1}{\ell}\right) \quad n=0,1,2, \ldots
$$

The case $n=0$ is just the normalization of $P_{\ell}$. Let us consider $n=1$,

$$
x P_{\ell}(x)=\frac{\ell+1}{2 \ell+1} P_{\ell+1}(x)+\frac{\ell}{2 \ell+1} P_{\ell-1}(x)
$$


[8, 9]. We notice that the coefficients on the right-hand side of (35) are positive, and that they add up to 1 , i.e., $x P_{\ell}(x)$ is a convex combination of $P_{\ell \pm 1}(x)$. It easily follows by induction that a similar relation holds for all $n$ and $\ell$,

$$
x^{n} P_{\ell}(x)=\sum_{k=-n}^{n}{ }^{\prime} a_{k}(n, \ell) P_{\ell+k}(x) ; \quad a_{k}(n, \ell)>0 ; \quad \sum_{k=-n}^{n}{ }^{\prime} a_{k}(n, \ell)=1,
$$

where the prime indicates that the sum runs only over $k$ with the same parity as $n$. Since the second of (36) is true for all $\ell$ and $n$, we must have,

$$
\lim _{\ell \rightarrow \infty} a_{k}(n, \ell)=a_{k}(n) \quad \text { with } \quad 0 \leq a_{k}(n) \leq 1
$$

and not all $a_{k}(n)=0$. Thus, we can write,

$$
\int_{-1}^{1} d x\left(x^{n} P_{\ell}(x)\right)^{2}=\int_{-1}^{1} d x \sum_{k, j=-n}^{n} a_{k}(n, \ell) a_{j}(n, \ell) P_{\ell+k}(x) P_{\ell+j}(x)=\sum_{k=-n}^{n} a_{k}^{2}(n, \ell) \frac{2}{2(\ell+k)+1}
$$

and, since $n$ and $k$ are independent of $\ell$, we can take the limit $\ell \rightarrow \infty$ to obtain,

$$
(2 \ell+1) \int_{-1}^{1}\left(x^{n} P_{\ell}(x)\right)^{2} \longrightarrow 2 \sum_{k=-n}^{n} a_{k}^{2}(n) .
$$

The same argument goes through for the term in (26) involving $Q_{\ell}$, because recursion relation (35) is valid if we substitute $Q_{\ell}$ for $P_{\ell}$ there, and because [8]

$$
\int_{-1}^{1} d x \mathrm{Q}_{\ell}^{2}(x)=\mathcal{O}\left(\frac{1}{\ell}\right) ; \quad \int_{-1}^{1} d x \mathrm{Q}_{\ell+n} \mathrm{Q}_{\ell+m}=\mathcal{O}\left(\frac{1}{\ell^{2}}\right) \quad(m \neq n)
$$

as $\ell \rightarrow \infty$. Therefore, $\left(\sigma_{2 \rightarrow 2}^{\ell}\right)_{A}$ is a finite constant in the limit $\ell \rightarrow \infty$ and we have,

$$
\left(\sigma_{2 \rightarrow 2}\right)_{A}=\lim _{L \rightarrow \infty} \sum_{\ell=0}^{L}\left(\sigma_{2 \rightarrow 2}^{\ell}\right)_{A}=\mathcal{O}(L)
$$

as claimed in the Introduction.

\subsection{The region of off-shell exchange}

We now turn to integral (25), which involves $Q_{\ell}$ and can be written in the form,

$$
\int_{1}^{\infty} d \xi \varphi(\xi)\left[\left(s+m_{X}^{2}\right)+\left(s-m_{X}^{2}\right) \xi\right] Q_{\ell}^{2}(\xi)
$$

where $\varphi$ represents the combination of factors in the integrand in (25), but we have explicitly extracted a term linear in $\xi$ coming from the denominator $\left(s-m_{Y_{*}}^{2}\right)$ in (25) which diverges at the upper limit of integration. We shall assume that $\varphi(\xi)$ is continuous and bounded in the interval of integration and that it is positive, and non-zero for $1 \leq \xi \leq a$, for some finite $a>1$. The integrand in (25) satisfies all of these conditions once the singular term $\left(s-m_{Y^{*}}^{2}\right)^{-1}$ is factored. First, we show that,

$$
\int_{a}^{\infty} d \xi \varphi(\xi)\left[\left(s+m_{X}^{2}\right)+\left(s-m_{X}^{2}\right) \xi\right] Q_{\ell}^{2}(\xi) \leq \frac{\mathrm{cst}}{a^{(2 \ell-1)} \ell} ; \quad a>1
$$


Notice that since $a>1$ the right-hand side of this equation is negligibly small for large $\ell$, and that better bounds can be found. We can write [8], for $\xi>1$,

$$
\begin{aligned}
Q_{\ell}(\xi) & =\sqrt{\frac{\pi}{2}} \frac{\left(\xi-\sqrt{\xi^{2}-1}\right)^{\ell+\frac{1}{2}}}{\left(\xi^{2}-1\right)^{\frac{1}{4}}} \frac{\Gamma(\ell+1)}{\Gamma\left(\ell+\frac{3}{2}\right)}{ }_{2} F_{1}\left(\frac{1}{2}, \frac{1}{2}, \ell+\frac{3}{2}, \frac{-\xi+\sqrt{\xi^{2}-1}}{2 \sqrt{\xi^{2}-1}}\right) \\
& \leq \sqrt{\frac{\pi}{2}} \frac{\left(\xi-\sqrt{\xi^{2}-1}\right)^{\ell+\frac{1}{2}}}{\left(\xi^{2}-1\right)^{\frac{1}{4}}} \frac{\Gamma(\ell+1)}{\Gamma\left(\ell+\frac{3}{2}\right)} \leq \sqrt{\frac{\pi}{2}} \frac{1}{\xi^{\ell+\frac{1}{2}}} \frac{1}{\left(\xi^{2}-1\right)^{\frac{1}{4}}} \frac{\Gamma(\ell+1)}{\Gamma\left(\ell+\frac{3}{2}\right)} .
\end{aligned}
$$

Substitution of this inequality in the left-hand side of (43) leads to the desired result, since $\varphi$ is bounded.

Next, we show that,

$$
\int_{1}^{a} d \xi \varphi(\xi)\left[\left(s+m_{X}^{2}\right)+\left(s-m_{X}^{2}\right) \xi\right] Q_{\ell}^{2}(\xi)=\mathcal{O}\left(\frac{1}{\ell^{2}}\right) \quad \text { as } \ell \rightarrow \infty .
$$

We can follow the same procedure as in the previous section, the only difference being that now odd powers of $\xi$ must also be considered. We have [8, 9],

$$
\int_{1}^{a} d \xi Q_{\ell}^{2}(\xi)=\int_{1}^{\infty} d \xi Q_{\ell}^{2}(\xi)-\int_{a}^{\infty} d \xi Q_{\ell}^{2}(\xi)=\frac{\psi^{\prime}(\ell+1)}{2 \ell+1}-o\left(\frac{1}{a^{2 \ell-1} \ell}\right) \rightarrow \frac{1}{\ell^{2}},
$$

where $\psi$ is a digamma function $[8,9]$. The case of even powers then follows from here since $Q_{\ell}$ satisfies the same recursion relation (35) as $P_{\ell}$, and since,

$$
\begin{aligned}
\int_{1}^{a} d \xi Q_{\ell+n}(\xi) Q_{\ell+m}(\xi) & =\int_{1}^{\infty} d \xi Q_{\ell+n}(\xi) Q_{\ell+m}(\xi)-\int_{a}^{\infty} d \xi Q_{\ell+n}(\xi) Q_{\ell+m}(\xi) \\
& =\frac{\psi(\ell+n+1)-\psi(\ell+m+1)}{(n-m)(2 \ell+n+m+1)}-o\left(\frac{1}{a^{2 \ell-1} \ell}\right) \rightarrow \frac{1}{\ell^{2}}
\end{aligned}
$$

We can then apply the same arguments as above. For odd powers we have, $\xi^{2 n} Q_{\ell}^{2}(\xi) \leq \xi^{2 n+1} Q_{\ell}^{2}(\xi) \leq$ $\xi^{2 n+2} Q_{\ell}^{2}(\xi)$ for $1 \leq \xi \leq a$ and hence the same result must hold. Finally,

$$
(2 \ell+1) \int_{1}^{\infty} d \xi \varphi(\xi)\left[\left(s+m_{X}^{2}\right)+\left(s-m_{X}^{2}\right) \xi\right] Q_{\ell}^{2}(\xi) \stackrel{\ell \rightarrow \infty}{\longrightarrow} \frac{\mathrm{cst}}{\ell}
$$

as asserted above.

\subsection{Remarks}

The preceding results show how the cross-section behaves as a function of $s$, and the influence of the kinematical cut $\lambda^{2}$. Let us take $\lambda^{2}<m_{Y}^{2}$, and consider $\left(\sigma_{2 \rightarrow 2}^{\ell}\right)_{A}$. For $s<m_{X}^{2}+\lambda^{2}$, $\left(\sigma_{2 \rightarrow 2}^{\ell}\right)_{A}=0$. When $m_{X}^{2}+\lambda^{2}<s<m_{X}^{2}+m_{Y}^{2}$, we have $\left(\sigma_{2 \rightarrow 2}^{\ell}\right)_{A}>0$ and, as shown in (41), $\left(\sigma_{2 \rightarrow 2}\right)_{A}$ diverges. Both $\left(\sigma_{2 \rightarrow 2}^{\ell}\right)_{A}$ and $\left(\sigma_{2 \rightarrow 2}\right)_{A}$ are of $\mathcal{O}\left(g^{6}\right)$, and therefore negligible in the 2-body approximation, because the integral in $\left(\sigma_{2 \rightarrow 2}^{\ell}\right)_{A}$ does not include the peak of $\Delta_{B W}\left(m_{Y^{*}}^{2}\right)$. It is only when $s-m_{X}^{2} \gtrsim m_{Y}^{2}$ that $\left(\sigma_{2 \rightarrow 2}^{\ell}\right)_{A},\left(\sigma_{2 \rightarrow 2}\right)_{A}$ are actually non-vanishing in the 2-body approximation. Thus, in this case, the precise value of $\lambda^{2}$ is irrelevant as long as it lies below the peak of $\Delta_{B W}$.

The cut does influence the dependence of $\left(\sigma_{2 \rightarrow 2}^{\ell}\right)_{B}$ on $s$. In the region $s<m_{X}^{2}+\lambda^{2}$, the branch point at $m_{Y^{*}}^{2}=s-m_{X}^{2}$ (or, $\xi=1$ ) is excluded by $\Theta$ (cuts) from the integration region in (25). 
Thus, (43) applies, implying that $\left(\sigma_{2 \rightarrow 2}^{\ell}\right)_{B}$ converges to zero fast enough to make $\left(\sigma_{2 \rightarrow 2}\right)_{B}$ finite. We are here essentially in the same situation as in (19) with $s<s_{-}$.

When $s>m_{X}^{2}+\lambda^{2}$, on the other hand, the range of integration in (25) extends all the way down to $m_{Y^{*}}^{2}=s-m_{X}^{2}$ (or, $\xi=1$ in (42)) and (48) holds, leading to a cross-section $\left(\sigma_{2 \rightarrow 2}\right)_{B}$ diverging with $L$ as $\log (L)$. For $s \gtrsim m_{X}^{2}+m_{Y}^{2}$, finally, the peak of $\Delta_{B W}\left(m_{Y^{*}}^{2}\right)$ leaves the integration region and $\left(\sigma_{2 \rightarrow 2}^{\ell}\right)_{B},\left(\sigma_{2 \rightarrow 2}\right)_{B}$ become $\mathcal{O}\left(g^{6}\right)$ and therefore negligible.

We see, then, that the value of $\lambda^{2}$ determines the point $s=m_{X}^{2}+\lambda^{2}$ at which $\left(\sigma_{2 \rightarrow 2}\right)_{B}$ is singular, i.e., it is the lowest value of $m_{Y^{*}}^{2}$ at which we start considering $Y$ to be "on-shell." It is therefore inherently ambiguous. In the case of (19), this value is taken as $m_{Y}^{2}$. When we take into account the finite width of $Y, \lambda^{2}$ should be set to $\lambda^{2}=m_{Y}^{2}-\alpha m_{Y} \Gamma_{Y}$ with $\alpha \sim 1-2$.

It is worth-while to stress here that the need for a cut $\lambda^{2}$ is independent of the kinematical singularity mentioned in the paragraph preceding eq. (28). Even if we set $m_{\phi}>0$, a cut in $m_{Y^{*}}^{2}$ is needed. Notice, however, that if $m_{\phi}>0$ we would also need an upper cut $\Theta\left(\lambda^{\prime 2}-m_{Y^{*}}^{2}\right)$, where now $\lambda^{\prime 2}=\lambda^{2}=m_{Y}^{2}+\alpha m_{Y} \Gamma_{Y}, \alpha \sim 1-2$. Since for $m_{\phi}=0$ the region of on-shell particle exchange extends to infinity, no upper kinematical cut is needed (or possible)-actually, $\Delta_{B W}\left(m_{Y^{*}}^{2}\right)$ does the job as described in the previous paragraph.

\section{$5 \quad$ Final Remarks}

In the foregoing sections we treated the problem of unstable-particle scattering in the context of 3 -body reactions. We showed how the cross-sections can be defined in sections 2 and 3 . The spatial singularities of the total cross-section were characterized in section 4 . The result found there for the total cross-section in the region of on-shell exchange, $\sigma_{2 \rightarrow 2}=\mathcal{O}(L)$, agrees with the conclusions of [10]. Indeed, an initial state consisting of wave-packets has an angular-momentum cut-off $L_{\max }$. Therefore, we must have in that case $\sigma_{2 \rightarrow 2} \sim L_{\max } \sim b$, with $b$ the largest available impact parameter, and then $\sigma_{2 \rightarrow 2}$ grows linearly with the transverse size of the wave-packets. In the general case of asymmetric wave-packets, of different radii in each beam, $\sigma_{2 \rightarrow 2}$ must be proportional to the largest one. In the region of off-shell exchange, $\sigma_{2 \rightarrow 2} \sim \log L$ as we approach the singular point.

The results of the previous sections were obtained at tree-level, with the exception of the BreitWigner form used for the propagators of unstable particles near their resonance peak. It is for this reason that we had to introduce an arbitrary mass cut-off $\lambda$ that separates the resonance peak, where the amplitude is $\mathcal{O}\left(g^{4}\right)$, from the tail where it is $\mathcal{O}\left(g^{6}\right)$. This cut $\lambda$ is inherently ambiguous, $\lambda^{2}=m^{2}-\alpha m \Gamma$ with $\alpha \sim 1-2$, and should not be necessary in a more complete treatment including loop corrections. We notice also that partial-wave cross-sections $\sigma_{2 \rightarrow 2}^{\ell}$ are of order $\sim g^{4} \log \left(\Gamma^{2} / m^{2}\right) /\left(m^{2} s^{2}\right)$ at their maximum. They are therefore far from saturating unitarity bounds as happens in the case of $s$-channel resonance formation, where $\sigma_{2 \rightarrow 2}^{\ell=0} \sim \mathcal{O}(1)$ in the coupling constant.

Let us consider one more time the Feynman graph for the process, redrawn in figure 3a for convenience. For $s_{-}<s<s_{+}$, the intermediate state indicated by the dashed line can be formed by particles 2, 3, 4 propagating on their mass-shell and forward in time. Given that particles 2 and 3 do interact, we ask what is the probability that these particles will propagate over a long distance before the interaction takes place. The heuristic Ansatz (29) implies that this probability decays exponentially with rate $1 / \Gamma_{5}$, the characteristic resonance formation time for particle 5 .

We have shown that at tree level there is no characteristic length-scale governing the process, 
thus leading to $\sigma_{2 \rightarrow 2}=\mathcal{O}(L)$ as $L \rightarrow \infty$. That result is most likely not changed by loop corrections when 1 and 5 in figure 3a are stable virtual particles. Whether the tree-level result is altered by loop corrections when unstable particles are involved and, in particular, whether loop corrections lead to (29) is currently under study. It is worth remarking here that equation (32) suggests that Ansatz (29) may not be the complete answer when more than one species of unstable particle are involved.

Self-energy loop insertions such as graph $3 \mathrm{~b}$ cannot change the basic conclusions reached at tree-level, although they lead to an amplitude with a stronger singularity $\sim 1 / u^{2}$, than that found at tree-level, $\sim 1 / u$. Resummation of these corrections, however, will only lead to mass and wavefunction renormalization, since particle 3 is stable and its self-energy cannot have an absorptive part at the pole. The opposite is true for vertex correction 3c, which will have a non-vanishing absorptive part when particle 3 is on-shell, but depends on $u$ as $\sim 1 / u$. As long as the coupling constant is small, this absorptive part should be a higher-order correction to the tree-level amplitude. We are then left with 1-particle irreducible graphs like 3d and 3e. Regarding those, we shall limit ourselves to point out that the unitary cut depicted in figure $3 \mathrm{f}$ leads directly to equation (18) for the cross-section. Notice that the extra loop in $3 \mathrm{f}$ is necessary [12], since the propagator of an unstable particle does not have a pole in the physical region.

\section{Acknowledgements}

I am indebted to Profs. V. Gupta and C.A. García Canal for their criticism of earlier versions of this paper and for suggesting many improvements. I also benefitted from a remarkably accurate referee's report.

I would like to thank Profs. J.M. Cornwall for pointing out ref. [6] to me, and G. López Castro for bringing ref. [2] to my attention and for several discussions.

I would also like to thank Profs. A. Zepeda Domínguez, M.A. Pérez Angón, A. García González and R. Huerta Quintanilla for help and support during these years at CINVESTAV.

This work has been partially supported by CONACYT through SNI and Research Project 0247P.

\section{References}

[1] R. Peierls, Phys. Rev. Let. 6, (1961), 641.

[2] I. Ginzburg, Nucl. Phys. Proc. Suppl. 51A,(1996),85; see also hep-ph/9509314.

[3] A. Bouzas, W. Y. Cheng and G. Gelmini, Phys. Rev. D55, (1997), 4663; see also hep$\mathrm{ph} / 9602278$.

[4] R. Suart, "Unstable Particles," in Proceedings of the Ringberg Workshop on Perspectives for Electroweak Interactions in $e^{+} e^{-}$Collisions, B. Kniehl editor, World Scientific, 1995.

[5] J. Bjorken, S. Drell, "Relativistic Quantum Fields," McGraw-Hill, New York, (1965).

[6] S. Coleman and R. Norton, Nuov. Cim. 38, (1965), 438.

[7] C. Itzykson and J.-B. Zuber, "Quantum Field Theory," McGraw-Hill, New York, (1980)

[8] A. Erdélyi et al., "Higher Trascendental Functions," McGraw-Hill, New York, 1953. 
[9] M. Abramowitz and I. Stegun, "Handbook of Mathematical Functions," Dover, New York, 9 edition.

[10] K. Melnikov and V. Serbo, Nucl. Phys. B483, (1997), 67.

[11] R. Wheeden and A. Zygmund, "Measure and Integral. An Introduction to Real Analysis," M. Dekker, New York, (1977).

[12] M. Veltman, Physica 29, (1963), 186.

\section{Figure Captions}

Figure 1 Generic Feynman diagrams for 3- and 2-body scattering processes.

Figure 2 3-body scattering graphs in the kinematical region where unstable internal particles are close to their pole mass.

Figure 3 (a)The graph in figure $2 \mathrm{a}$ is redrawn here omitting the external legs labelled $p_{1-4}$. Particles 1 and 5 correspond to $X, Y$ and 2,3,4 to $\phi$, resp., in fig. 2a. (b)-(e) Some one-loop corrections to the process depicted in (a). (f) A unitary cut in (d). 


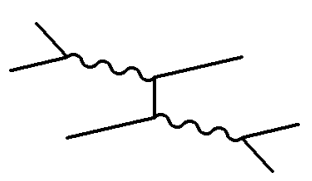

(a)

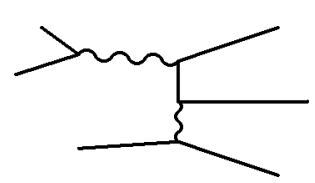

(b)

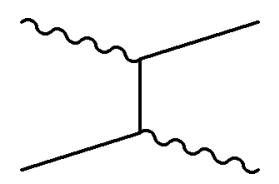

(c)

Figure 1:

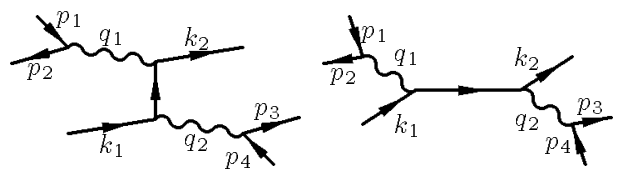

(a)

(b)

Figure 2:

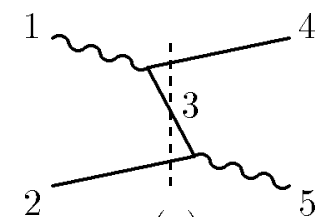

(a)

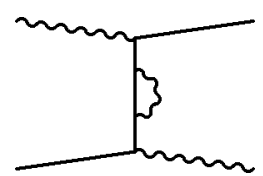

(b)

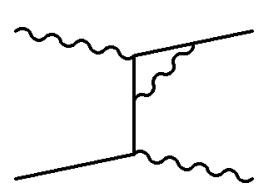

(c)

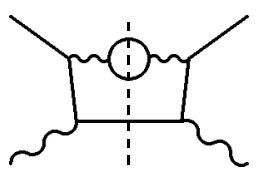

(f)

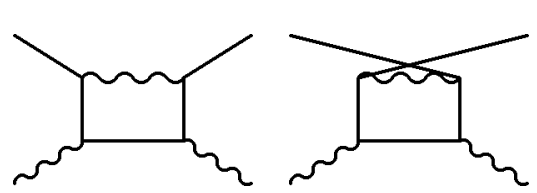

(d)

(e)

Figure 3: 